\title{
A MINIMUM CURVATURE COMBINATION METHOD FOR THE GENERATION OF MULTI-PLATFORM DINSAR DEFORMATION TIME-SERIES
}

\author{
Pepe A. ${ }^{(1)}$, Solaro G. ${ }^{(1)}$, Dema C. ${ }^{(2)}$ \\ (I) Istituto per il Rilevamento Elettromagnetico dell'Ambiente - Consiglio Nazionale delle Ricerche (CNR) \\ Via Diocleziano, 328 - 80124 Napoli (Italy),Email: \{pepe.a;solaro.g\}@irea.cnr.it \\ (2) Università degli Studi della Basilicata, viale dell'Ateneo Lucano,10,85100 Potenza,Email: claudio.dema92@gmail.com
}

\begin{abstract}
We present a DInSAR approach for the retrieval of the deformation components through a multi-angle/multiplatform combination strategy. A minimum-curvature combination method has been adopted, based on the exploitation of the line-of-sight (LOS) displacement time-series retrieved by independently processed sequences of SAR data frames. The proposed method can also be seen as a further extension of the MSBAS DInSAR approach but, at variance with it, the algorithm does not require the simultaneous processing of the SAR multi-platform data-sets, and can be applied with no restrictions on the method used for the retrieval of LOS DInSAR time-series, making the field of applicability of it extremely wide. Tests conducted on simulated and real data confirm the validity of the proposed approach. Experiments on real data have been carried out by analyzing three different SAR datasets collected from 2003 to 2010 by the ASAR/ENVISAT (on ascending and descending passes) and the ALOS sensors, respectively.
\end{abstract}

\section{INTRODUCTION}

Differential Synthetic Aperture Radar Interferometry (DInSAR) technique [1] for the monitoring of Earth surface deformation has evolved over the years to represent nowadays a powerful tool in scientific and operational contexts. Historically developed to study single deformation episodes, DInSAR is now mostly used to follow the temporal evolution of surface deformation through the generation of displacement time-series, retrieved by applying advanced multitemporal DInSAR approaches [3]. These methods are based on the inversion of properly generated sequences of DInSAR interferograms, and allow the computation of Line Of Sight (LOS)-projected deformation timeseries in areas that are not significantly affected by decorrelation noise artifacts [2].

At the present days, the growing availability of large archives of SAR images collected by several sensors, mounted onboard to constellations of space-based platforms operating at different wavelengths, and with complementary looking angle geometries (and also possibly through different acquisition modes) poses the problem to effectively combine the pieces of information coming from them. In particular, the combination of multi-platform/multi-looking angle DInSAR-based displacement measurements can improve our ability in mapping surface deformation in three dimension, thus overcoming the inherent limitation of InSAR to exclusively measure the sensor's LOS projection of the deformation. Noteworthy, all modern space-based SAR platforms are in a quite nearpolar orbit and acquire images over ascending and descending passes; accordingly, the ability to discriminate the north-south components of deformation is quite limited. However, the combination of ascending/descending mean displacement velocity maps is still possible [5], and allows retrieving the rate of deformation w.r.t. Up-Down and East-West directions, respectively. More challenging is the problem to recover, not only, the Up-Down and East-West mean displacement rate maps, but also to follow the temporal evolution of both deformations components by retrieving, hence, the Up-Down and East-West timeseries for the overlapped time between ascending and descending passes. This problem has already been faced in literature and a few solutions have been proposed in recent years [6]-[7]. In particular, the algorithm presented in [6] and known as MSBAS, was designed as an extension of the Small BAseline Subset (SBAS) method [4] and consists in acquiring, and simultaneously processing, a very large sequence of multiple-platform interferograms, which are then inverted to recover the (2-D) displacement time-series by using a regularized Singular Value Decomposition (SVD)-based approach.

In our work, we investigate the potential of an alternative solution, which does not require the simultaneous process of large archives of DInSAR interferograms, and consists in the development of a simpler post-processing step to be carried out on sets of already independently processed (potentially, with different DInSAR processing tools) of multi-sensor LOS displacement time-series, preliminarily represented in a common geocoded grid. The presented method 


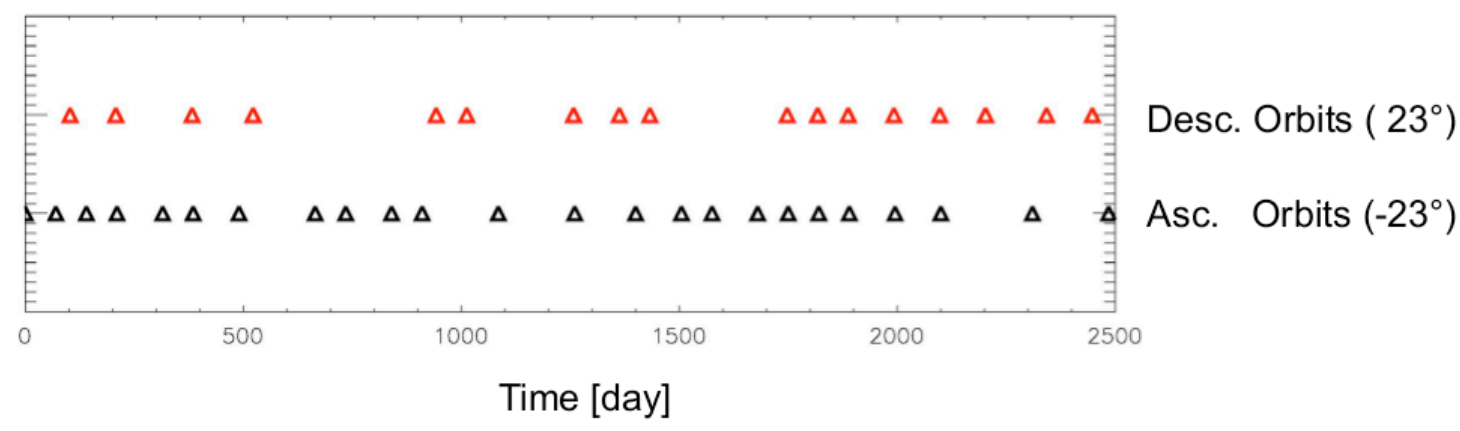

Figure 1. Time Acquisitions of the simulated data sets used for experimental analyses.

relies on a minimum-curvature-based combination approach, firstly adopted in [8] for the generation of LOS deformation time-series and here properly adapted to the problem at hand. The algorithm has specifically been developed to work in the SBAS framework. However the adopted scheme can be used in a generalpurpose PS- and SB-oriented context.

The following section will provide a description of the method from a theoretical point of view, whereas in Section III we present the results of a series of experiments that have been conducted both on simulated and real data.

\section{THEORY}

Let us suppose the availability of $\mathrm{K}$ sets of independently retrieved (by applying either the SBAS approach or other alternative DInSAR techniques) geocoded LOS-projected DInSAR deformation timeseries, namely $\mathbf{d}^{(\mathbf{j})} \equiv\left\lfloor d_{1}^{(j)}-d_{0}^{(j)}, d_{2}^{(j)}-d_{0}^{(j)} \ldots, d_{N_{j}-1}^{(j)}-d_{0}^{(j)}\right\rfloor^{T}$, which are relevant to time vectors $\mathbf{t}^{(j)} \equiv\left\lfloor t_{0}^{(j)}, t_{1}^{(j)}, \ldots, t_{N_{j}}^{(j)}\right\rfloor \quad j=1, \ldots, K \quad$ consisting of $N_{j}$ distinctive time acquisitions and are calculated relative to the deformation $d_{0}^{(j)}$ associated to the first time epoch $t_{0}^{(j)}$ of each single SAR data set. By considering the general guidelines of [4],[6], for each pixel of the (geocoded) grid, a system of $N=\sum_{j=1}^{K}\left(N_{j}-1\right)$ independent linear equations with respect to $M=3 \cdot\left[\left(\sum_{j=1}^{K} N_{j}\right)-1\right]$ unknowns can be written, where the unknowns $\quad V_{U P}=\left[V_{U P}\left(T_{1}\right), \ldots, V_{U P}\left(T_{P-1}\right)\right]^{T}$, $V_{E A S T}=\left[V_{E A S T}\left(T_{1}\right), \ldots, V_{E A S T}\left(T_{P-1}\right)\right]^{T}, V_{N}=\left[V_{N}\left(T_{1}\right), \ldots, V_{N}\left(T_{P-1}\right)\right]^{T}$ and $\mathrm{X}$ is the generic component of the deformation signal. More precisely, the system assumes the following form:

$$
\mathbf{B} \cdot\left(\begin{array}{c}
\mathbf{V}_{\mathbf{E A S T}} \\
\mathbf{V}_{\mathbf{U P}} \\
\mathbf{V}_{\mathbf{N}}
\end{array}\right)=\left(\begin{array}{c}
\mathbf{d}^{(1)} \\
\ldots \\
\mathbf{d}^{(K)}
\end{array}\right)(1 \mathrm{a})
$$

with:

$$
\mathbf{B} \equiv\left[\begin{array}{ccc}
\mathbf{B}^{(1)} \sin \theta^{(1)} \cos \phi^{(1)} & \mathbf{B}^{(1)} \cos \theta^{(1)} & \mathbf{B}^{(1)} \sin \theta^{(1)} \sin \varphi^{(1)} \\
\mathbf{B}^{(2)} \sin \theta^{(2)} \cos \phi^{(2)} & \mathbf{B}^{(2)} \cos \theta^{(2)} & \mathbf{B}^{(2)} \sin \theta^{(2)} \sin \varphi^{(2)} \\
\ldots & \ldots & \ldots \\
\ldots & \ldots & \ldots \\
\mathbf{B}^{(\mathbf{K})} \sin \theta^{(K)} \cos \phi^{(K)} & \mathbf{B}^{(\mathbf{K})} \cos \theta^{(K)} & \mathbf{B}^{(\mathbf{K})} \sin \theta^{(K)} \sin \varphi^{(K)}
\end{array}\right]
$$

being the incidence-like matrix of the applied linear transformations (see [5] for more details) that relate the $\mathrm{M}$ unknowns to the available $\mathrm{K}$ sets of LOS deformation time-series. Note that $\left[\theta^{(1)}, \theta^{(2)}, \ldots, \theta^{(K)}\right]^{T}$ and $\left[\varphi^{(1)}, \varphi^{(2)}, \ldots, \varphi^{(K)}\right]^{T}$ are the sensor side looking angles and inclinations angles of the sensor flight orbit with respect to the North-South direction, respectively. However, all modern space-borne satellites orbit the Earth following near-polar trajectories, hence sensor orbits are almost parallel to the North-South directions and inclination angles are small (around $10^{\circ}$ ). Accordingly, sensibility of DInSAR measurement with respect to North-South direction is very low and, as a consequence, Eq. (1) can be well approximated as:

$$
\hat{\mathbf{B}} \cdot\left(\begin{array}{c}
\mathbf{V}_{\text {EAST }} \\
\mathbf{V}_{\mathbf{U P}}
\end{array}\right)=\left(\begin{array}{c}
\mathbf{d}^{(1)} \\
\ldots \\
\mathbf{d}^{(K)}
\end{array}\right)(2)
$$

where $\hat{\mathbf{B}}=\left.\mathbf{B}\right|_{\varphi^{(k)}=0} \forall k=1, \ldots, K$. The system (2) is under-determined and hence does not admit a unique solution. Similarly to [6] this system can be regularized by adding a set of additional equations; however, in our case, the regularization is achieved by imposing the 


\section{UP-DOWN}
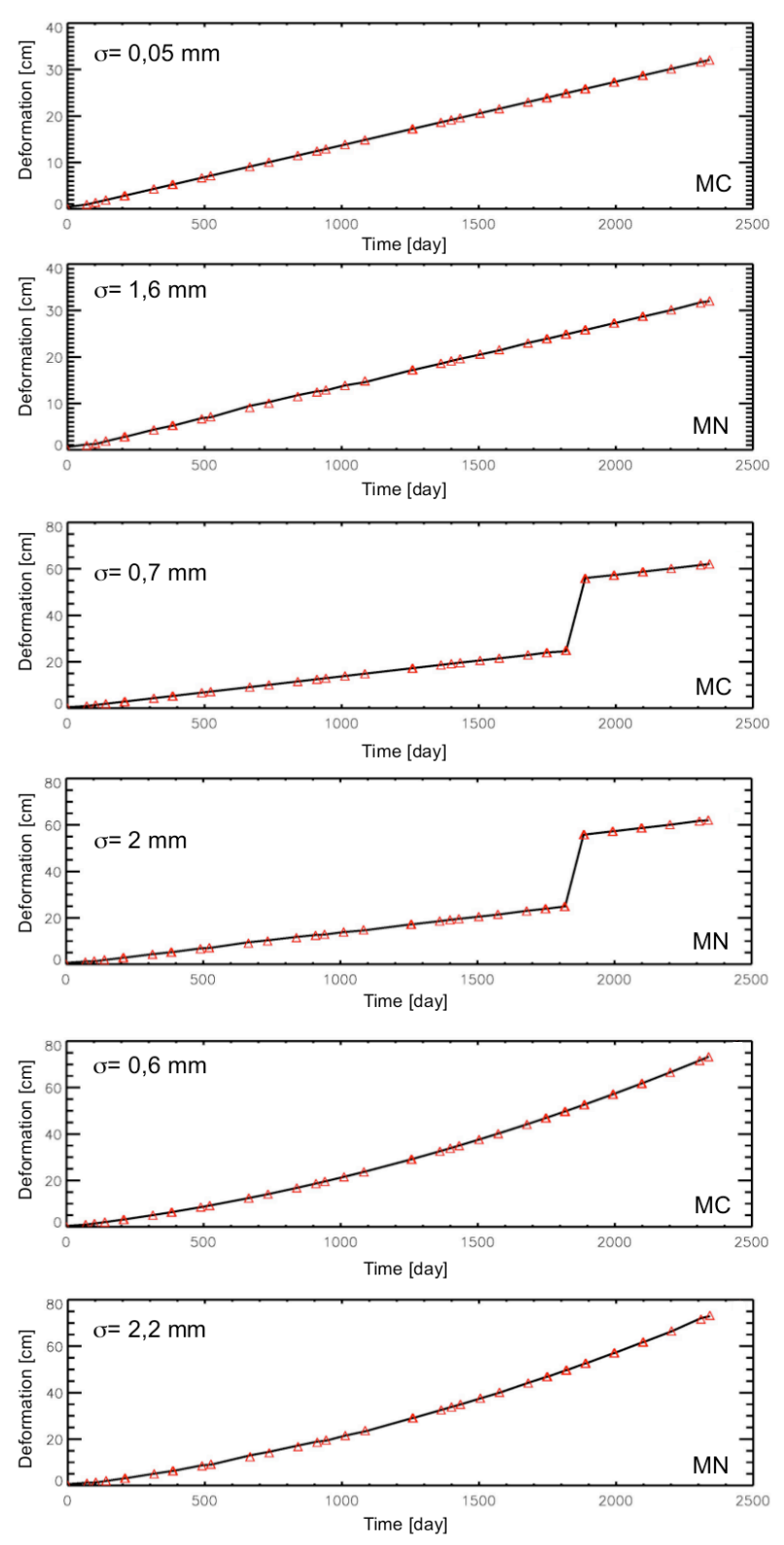

EAST-WEST
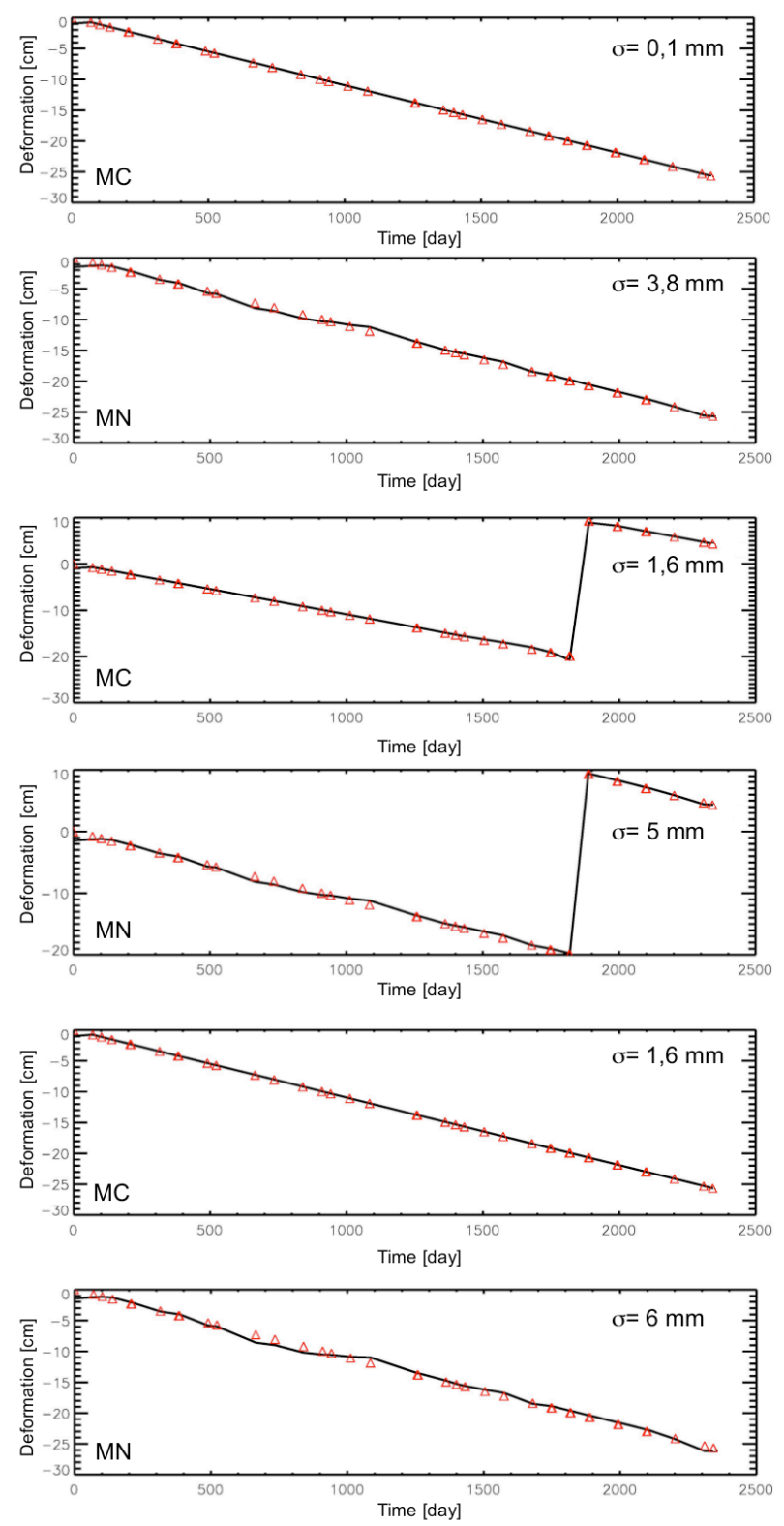

Figure 2. Comparison between synthesized (red triangles) and retrieved (black lines) Up-Down (left side) and East-West (right side) time-series of deformation. Comparisons between the Minimum-Curvature (MC) and Minimum-Norm (MN) solutions are also provided. The standard deviation values of the difference between simulated and obtained displacement signals have also been computed and shown in the figure. 


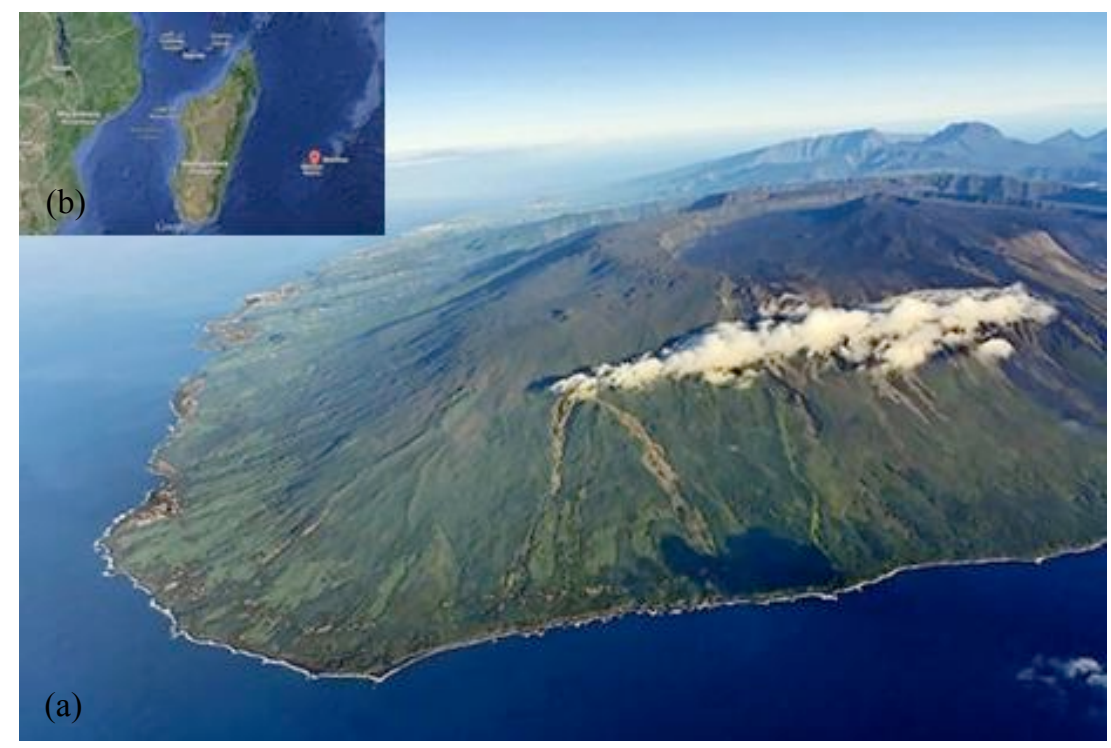

Figure 3. (a) Piton de la Fournaise and (b) its geographical location.

condition that the East-West and Up-Down time-series are with minimum curvature, that is with minimal acceleration between consecutive time intervals: $\left(V_{U P}\left(T_{i+1}\right)-V_{U P}\left(T_{i}\right)\right) /\left(T_{i+1}-T_{i-1}\right)=0$, $\left(V_{E A S T}\left(T_{i+1}\right)-V_{E A S T}\left(T_{i}\right)\right) /\left(T_{i+1}-T_{i-1}\right)=0$. Accordingly, the system that has to be solved becomes:

$$
\left(\begin{array}{c}
\hat{\mathbf{B}} \\
\lambda \cdot \mathbf{C}
\end{array}\right) \cdot\left(\begin{array}{c}
\mathbf{V}_{\text {EAST }} \\
\mathbf{V}_{\mathbf{U P}}
\end{array}\right)=\left(\begin{array}{c}
\mathbf{d}^{(\mathbf{1})} \\
\ldots \\
\mathbf{d}^{(\mathbf{K})} \\
\mathbf{0}
\end{array}\right)
$$

where $\lambda$ is a regularization parameter, at variance with [6] where the regularization was done by minimizing the norm of the velocity. The solution of (3) is obtained in a LS sense through the SVD.

The main advantages in using the proposed combination scheme with respect to previous solutions arise in: i) the possibility to use "conventional" processing tools for the retrieval of LOS displacement maps, thus also making it possible the combination of DInSAR displacement time-series produced by different, and inherently complementary, InSAR tools; ii) the possibility to integrate in the post-processing "minimum curvature" combination scheme information on the quality of reconstruction of the deformation (e.g. the information on the temporal coherence of investigated pixels [9]) as weighting factors; iii) the possibility to complement DInSAR-based deformation measurements with other external sources of information (GPS/leveling data); iv) its ability to be simply adapted to "update" previously achieved 2-D displacement timeseries as soon as new SAR acquisitions are available: this is particularly important in the light of new SAR systems, such as the Sentinel-1 SAR platform, planned to collect data with a only few days repetition frequency.

\section{EXPERIMENTAL RESULTS}

To validate the proposed approach a series of experiments have been performed on simulated and real data. We start our analysis by first considering a simulated scenario. In particular, we assume the availability of two synthesized LOS displacement timeseries, namely $\boldsymbol{d}_{A S C}\left(\boldsymbol{t}_{A S C}\right)$ and $\boldsymbol{d}_{D E S C}\left(\boldsymbol{t}_{D E S C}\right)$, computed by assuming certain East-West/Up-Down deformation signals on the ground as seen by two radar sensors (e.g., we considered for the simulation the radar and geometrical parameters of the ASAR/ENVISAT sensor) flying over the scene along ascending and descending passes, at the time epochs $\boldsymbol{t}_{A S C}$ and $\boldsymbol{t}_{D E S C}$, identified by red and black triangles, respectively, in Figure 1. Figure 2 summarizes the results of a cross-comparison analysis between the simulated (black line) and the retrieved East-West and Up-Down deformation time-series for three specific simulated scenarios consisting in: i) E-W and Up-Down linear deformation trends, ii) linear displacement trend abruptly interrupted by jumps (likely due to earthquakes), and iii) quadratic deformation signals. The results for both the minimum-curvature (MC) and minimum-norm (MN) solutions are presented. As expected, they clearly evidence that when deformations are linear (or quasi-linear) and/or quadratic, $\mathrm{MC}$ regularization is definitely better than the $\mathrm{MC}$ one, but also in the presence of jumps MC regularization looks adequate, enough. This is proved by the estimated standard deviations differences that, even though simulated signals are corrupted by noise, are of the order of a few millimeters. However, for a comprehensive evaluation of the MC performances additional tests are needed to quantify the impact of the 

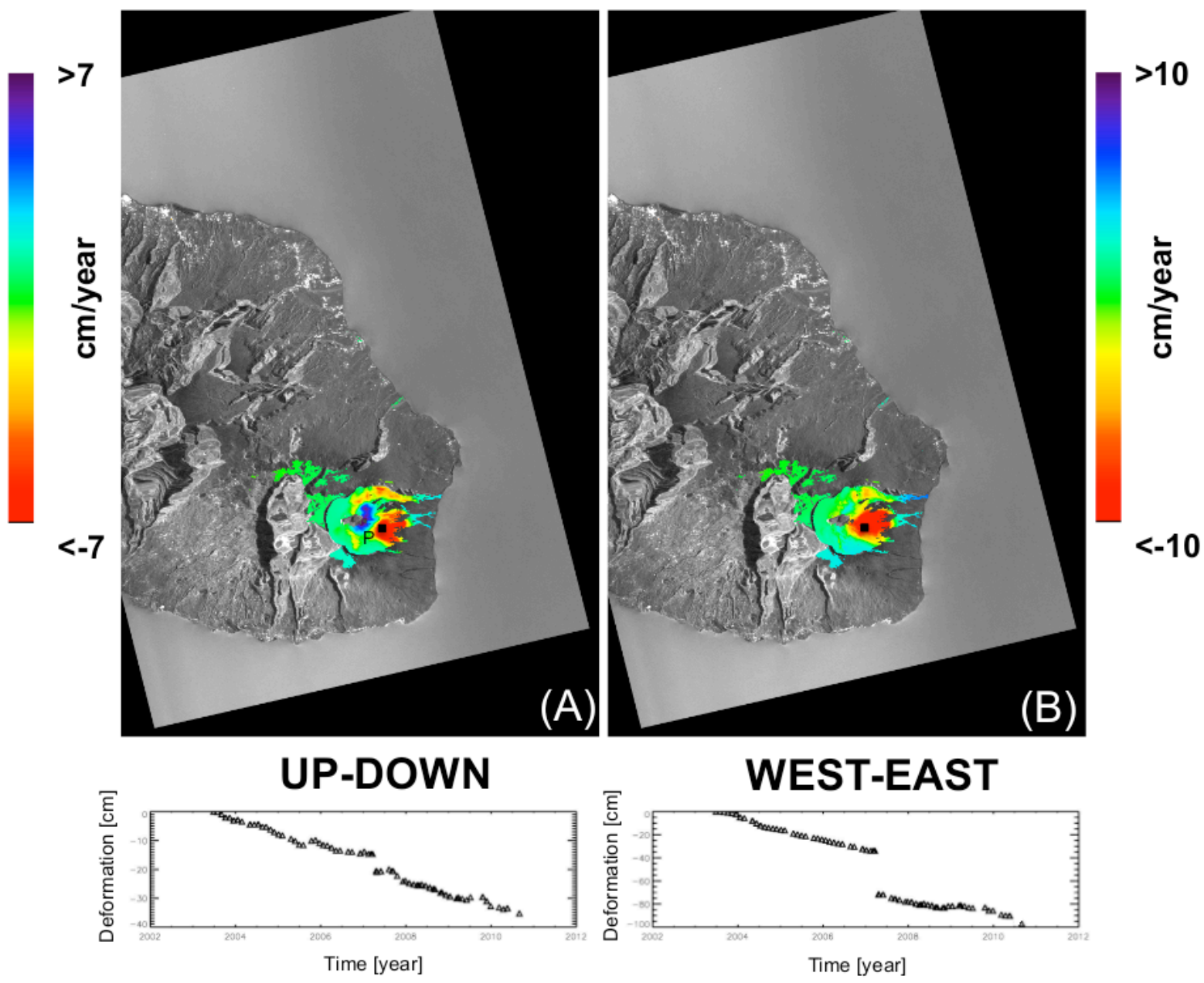

Figure 4. DInSAR results retrieved over Piton de La Fournaise area. (a) Up-Down and (b) mean velocity displacement maps of the area. Plots show the Up-Down (left side) and East-West (right side) deformation signals captured in correspondence to the pixel P highlighted by a black square in (a).

number and time distribution of SAR measurements as well as the noise on achievable results; this is matter for future and extensive analyses.

Finally, we have also carried out an experiment on real data by focusing on the case-study area of Piton La Fournaise (Reunion Islands), see Figure 3, which is home of a large volcanic system that recently erupted recently (on April 3, 2007), leading to large fractures on the ground. This volcanic system has extensively been studied over recent years. For our purposes, we exploited three independent sets of SAR acquisitions collected by the ENVISAT/ASAR (C-band) instrument along ascending (48 images) and descending passes (35 images) as well as by the ALOS-1/PALSAR (L-band) sensor (11 images) over a time period spanning the 2007 eruption. These three sets of SAR data were independently processed by the SBAS-DInSAR processing chain, leading to the generation of corresponding LOS-projected displacement time-series, which have then been geocoded with respect to a common SAR geometry. For all the pixels that have remained coherent in all the three independent DInSAR processing analyses, we have applied the proposed minimum curvature combination method (in a postprocessing phase), which lead us to discriminate the time-series of the deformation components. Figure 4 shows the maps of retrieved East-West and Up-Down mean deformation velocity signals, superimposed to greyscale SAR amplitude images of the zone; note that in these maps only pixels for which temporal coherence was greater than a given threshold (we used a value of $0.6)$ have been portrayed.

A point located in the summit area of the rater was also selected and the inherent E-W and Up-Down deformation time-series have shown in the plots of Figure3. They make it evident the large E-W movement of the crust due to the eruption (around $40 \mathrm{~cm}$ ) that moved seawards; also a significant Up-Down signal was present even with more moderate deformation values (around $8 \mathrm{~cm}$ ). 


\begin{tabular}{|c|c|c|c|}
\hline Dav & Month & Year & Perpendicular Baseline \\
\hline 24 & 6 & 2003 & -112 \\
\hline 29 & 7 & 2003 & 124,3 \\
\hline 2 & 9 & 2003 & $-417,8$ \\
\hline 7 & 10 & 2003 & $-134,2$ \\
\hline 11 & 11 & 2003 & 760,1 \\
\hline 16 & 12 & 2003 & 903,8 \\
\hline 20 & 1 & 2004 & 653,7 \\
\hline 24 & 2 & 2004 & 957,5 \\
\hline 4 & 5 & 2004 & 774,9 \\
\hline 8 & 6 & 2004 & $-529,1$ \\
\hline 13 & 7 & 2004 & $-23,2$ \\
\hline 17 & 8 & 2004 & 772,5 \\
\hline 26 & 10 & 2004 & $-673,4$ \\
\hline 4 & 1 & 2005 & 1,4 \\
\hline 19 & 4 & 2005 & $-528,1$ \\
\hline 24 & 5 & 2005 & $-52,9$ \\
\hline 28 & 6 & 2005 & $-714,8$ \\
\hline 2 & 8 & 2005 & 46,1 \\
\hline 11 & 10 & 2005 & $-21,7$ \\
\hline 15 & 11 & 2005 & $-371,3$ \\
\hline 20 & 12 & 2005 & $-50,6$ \\
\hline 9 & 5 & 2006 & $-180,1$ \\
\hline 13 & 6 & 2006 & 0 \\
\hline 22 & 8 & 2006 & $-696,8$ \\
\hline 5 & 12 & 2006 & 17,6 \\
\hline 13 & 2 & 2007 & 77,7 \\
\hline 24 & 2 & 2007 & 21,5 \\
\hline 29 & 5 & 2007 & $-46,2$ \\
\hline 7 & 8 & 2007 & 121,9 \\
\hline 11 & 9 & 2007 & $-107,8$ \\
\hline 16 & 10 & 2007 & 103 \\
\hline 25 & 12 & 2007 & 255,8 \\
\hline 29 & 1 & 2008 & $-14,5$ \\
\hline 4 & 3 & 2008 & 330,5 \\
\hline 8 & 4 & 2008 & $-211,9$ \\
\hline 13 & 5 & 2008 & $-7,2$ \\
\hline 17 & 6 & 2008 & $-169,2$ \\
\hline 22 & 7 & 2008 & -160 \\
\hline 26 & 8 & 2008 & -8 \\
\hline 4 & 11 & 2008 & 27,2 \\
\hline 9 & 12 & 2008 & 189,6 \\
\hline 13 & 1 & 2009 & $-116,7$ \\
\hline 24 & 3 & 2009 & $-367,5$ \\
\hline 2 & 6 & 2009 & $-225,2$ \\
\hline 20 & 10 & 2009 & 153 \\
\hline 24 & 11 & 2009 & -191 \\
\hline 13 & 4 & 2010 & $-96,8$ \\
\hline 18 & 5 & 2010 & $-188,4$ \\
\hline
\end{tabular}

\begin{tabular}{|c|c|c|c|}
\hline Day & Month & Year & Perpendicular Baseline \\
\hline 5 & 10 & 2003 & $-365,5$ \\
\hline 14 & 12 & 2003 & $-735,1$ \\
\hline 18 & 1 & 2004 & $-608,4$ \\
\hline 2 & 5 & 2004 & -218 \\
\hline 11 & 7 & 2004 & $-107,8$ \\
\hline 19 & 9 & 2004 & $-246,6$ \\
\hline 28 & 11 & 2004 & $-37,5$ \\
\hline 6 & 2 & 2005 & $-122,5$ \\
\hline 22 & 1 & 2006 & $-546,9$ \\
\hline 26 & 2 & 2006 & $-492,5$ \\
\hline 2 & 4 & 2006 & $-205,8$ \\
\hline 24 & 9 & 2006 & 0 \\
\hline 3 & 12 & 2006 & $-215,1$ \\
\hline 7 & 1 & 2007 & $-270,1$ \\
\hline 18 & 3 & 2007 & -130 \\
\hline 22 & 4 & 2007 & 107,6 \\
\hline 27 & 5 & 2007 & 54,2 \\
\hline 27 & 1 & 2008 & -236 \\
\hline 6 & 4 & 2008 & $-213,7$ \\
\hline 11 & 5 & 2008 & 6,5 \\
\hline 15 & 6 & 2008 & 100,5 \\
\hline 20 & 7 & 2008 & 57,8 \\
\hline 24 & 8 & 2008 & $-360,4$ \\
\hline 2 & 11 & 2008 & $-273,8$ \\
\hline 7 & 12 & 2008 & 148,3 \\
\hline 11 & 1 & 2009 & $-117,7$ \\
\hline 22 & 3 & 2009 & $-138,7$ \\
\hline 26 & 4 & 2009 & 182,7 \\
\hline 5 & 7 & 2009 & $-74,3$ \\
\hline 18 & 10 & 2009 & $-57,4$ \\
\hline 22 & 11 & 2009 & $-113,7$ \\
\hline 27 & 12 & 2009 & 169,2 \\
\hline 7 & 3 & 2010 & $-141,1$ \\
\hline 16 & 5 & 2010 & 134,3 \\
\hline 29 & 8 & 2010 & $-258,5$ \\
\hline
\end{tabular}

(a)

(b)

Table 1. Ascending (a) and Descending (b) ENVISAT/ASAR data set used in the presented experiments.

\begin{tabular}{cccc}
\hline Day & Month & Year & Perpendicular Baseline \\
\hline 4 & 3 & 2007 & 0 \\
19 & 4 & 2007 & 36,5 \\
4 & 9 & 2007 & $-308,7$ \\
5 & 12 & 2007 & -892 \\
6 & 3 & 2008 & $-1316,7$ \\
21 & 4 & 2008 & $-1370,7$ \\
22 & 7 & 2008 & $-38,5$ \\
6 & 9 & 2008 & 1261 \\
22 & 10 & 2008 & 1412,4 \\
9 & 3 & 2009 & 617,3 \\
28 & 7 & 2010 & -1038 \\
\hline
\end{tabular}

Table 1I. ALOS-1 PALSAR SAR data set used in the presented experiments.

\section{ACKNOWLEDMENTS}

This work has partly been funded by the European Commission under the MED-SUV project (European
Union's Seventh Programme for research, technological development and demotrastion under grant agreement No. 308665).

The authors would like to thank the European Space Agency for providing the ENVISAT ASAR data and the 
Japanese Space Agency (JAXA) that has provided the ALOS-1 data through the project entitled "Advanced Interferometric SAR Techniques for Earth Observation at L-band" (ID project 1149) in the framework of "The 4-th ALOS Research Announcement for ALOS-2" call. They also thank University of Delft that provided precise orbital information. The DEM of the investigated zone was acquired through the SRTM archive. Finally, the authors are grateful to S. Guarino, M. Rasulo and F. Parisi for their technical support.

\section{REFERENCES}

[1] R. Bürgmann, P. A. Rosen, and E. J. Fielding, "Synthetic aperture radar interferometry to measure Earth's surface topography and its deformation," Annu. Rev. Earth Planet. Sci., vol. 28, pp. 169-209, May 2000.

[2] H. A. Zebker and J. Villasenor, "Decorrelation in interferometric radar echoes," IEEE Trans. Geosci. Remote Sens., vol. 30, pp. 950-959, Sep. 1992.

[3] A. Ferretti, C. Prati, and F. Rocca, "Permanent scatterers in SAR interferometry," IEEE Trans. Geosci. Remote Sens., vol. 39, no. 1, pp. 8-20, Jan. 2001.

[4] P. Berardino, G. Fornaro, R. Lanari, and E. Sansosti, "A new algorithm for surface deformation monitoring based on small baseline differential SAR interferograms," IEEE Trans. Geosci. Remote Sens., vol. 40, no. 11, pp. 2375-2383, Nov. 2002.

[5] M. Manzo, Ricciardi, G. P., Casu, F., Ventura, G., Zeni, G., Borgström, S., Berardino, P., Del Gaudio, C., and Lanari, R., "Surface deformation analysis in the Ischia island (Italy) based on spaceborne radar interferometry", Journal of Volcanology and Geothermal Research, 151, pp. 399-416, 2006.

[6] S. Samsonov, N. d'Oreye , "Multidimensional timeseries analysis of ground deformation from multiple InSAR data sets applied to Virunga Volcanic Province", Geophysical Journal International 191, pp. 1095-1108, 2012.

[7] J. Hu, X. L. Ding, Z. W. Li, J. J. Zhu, Q. Sun, and L. Zhang, "Kalman-Filter-Based Approach for Multisensor,Multitrack, and Multitemporal InSAR", IEEE Trans. Geosc. Remote Sens., vol. 51, no. 7, July 2013.

[8] M. Costantini, F. Miniati and L. Pietranera, "Combining Multi-Temporal SAR Differential Interferograms: A Curvature based method",
Fringe Symposium, Frascati, 2004.

[9] A. Pepe, and R. Lanari, "On the extension of the minimum cost flow algorithm for phase unwrapping of multitemporal differential SAR interferograms," IEEE Trans. Geosci. Remote Sens., vol. 44, no. 9, pp. 2374-2383, Sept. 2006. 